\title{
Effect of modern radiation techniques on the surgery of nonmalignant intracranial tumors
}

\author{
Roberto C. Heros, M.D. \\ Department of Neurosurgery, University of Miami, Florida
}

$\mathrm{I}$ WAS ASKED by Dr. Johnson, the editor of this issue of Neurosurgical Focus, to write an editorial describing how current radiation techniques may have influenced my surgical attitude toward nonmalignant intracranial tumors. I am delighted to have been given this opportunity and would like to begin with 2 disclaimers.

The first disclaimer is that I personally do not perform radiosurgery in any of its forms. Obviously, I am not an expert either in any of the modern forms of fractionated radiotherapy. Therefore, I will not discuss specific forms of delivery of radiation, advantages of one form of radiation over another, or radiation dosing.

The second important disclaimer is that I have never engaged in scientific investigation concerning benign intracranial tumors and practically none of my writings have addressed this topic. Furthermore, until about 13 or 14 years ago, although I was always interested in the surgery of specific benign intracranial tumors, such as craniopharyngiomas and some pineal tumors in particular, I operated on benign intracranial tumors only sporadically and $75-80 \%$ of my practice consisted of neurovascular surgery. However, with the advent of radiosurgery for arteriovenous malformations, endovascular surgery for aneurysms, a greater number of well-trained neurovascular surgeons competing for these cases, and other reasons, the volume of my neurovascular practice has gradually declined. As a consequence, I have become more and more engaged in the surgery of benign intracranial tumors to the point that, at the present time, that aspect of my practice exceeds the volume of my neurovascular cases. The experience acquired in skull base exposures for vascular lesions helped considerably as my practice with benign tumors of the base of the skull increased.

I would like to organize my comments by addressing specific intracranial locations of tumors and then, in a very general way, discuss how modern radiation techniques may have influenced my current surgical attitude towards tumors in these different locations.

\section{Cerebral Convexity Tumors}

In this section on cerebral convexity, I will refer only to meningiomas. I believe that the most important decision with meningiomas of the convexity is not how to remove them, but when. I tend to see many more asymptomatic patients with convexity meningiomas in my clinic than patients with truly symptomatic meningiomas. The decision to operate is easy in clearly symptomatic patients or in patients with relatively large meningiomas with significant mass effect. I tend to use the presence of significant edema, even if the tumor itself is not too large, as an indication for surgery, but I know that the evidence supporting this policy is meager to nonexistent. Not infrequently, I would recommend surgery for an asymptomatic tumor-even if it is not producing significant mass effect-when the tumor is close to a major venous sinus (including the cavernous sinus) under the presumption that if the tumor is allowed to grow to the point of involving the sinus, the surgery may become riskier at that time and the possibility of a "cure" decreases substantially. In general, if I recommend treatment for a convexity meningioma, that treatment is excision, and only rarely would I recommend radiosurgery unless the patient is elderly or has substantial surgical comorbidities. With atypical meningiomas that have been removed completely, including their dural attachments, I prefer observation because the evidence for the effectiveness of radiosurgery in this setting is inconclusive. ${ }^{1}$ For the occasional malignant meningioma, I recommend, as I suspect do most neurosurgeons, postoperative fractionated radiotherapy with a relatively wide margin around the tumor. This is also my recommendation for most instances of recurrent tumor given the propensity for second recurrences to occur more commonly and more rapidly. ${ }^{2}$

\section{Parasagittal Tumors}

In discussing parasagittal tumors, we are again usually talking about meningiomas. There is little controversy about surgery for meningiomas involving the anterior third of the sagittal sinus, which can generally be removed radically, including resection of the sinus and falx when necessary. More posterior parasagittal meningiomas_-particularly those involving the middle third of the sagittal sinus - are more problematic, and I recently had the opportunity to write an editorial commenting on the excellent article by Sindou and Alvernia ${ }^{6}$ reviewing their outstanding experience with the surgery of these tumors. ${ }^{4}$ Essentially, my point in that editorial was that I personally, not pos- 
sessing the technical skill and experience of Dr. Sindou with tumors in this location, have a more conservative attitude toward tumors that do not completely occlude the sinus. Rather than removing these tumors radically and either exploring or reconstructing the sinus, as performed so skillfully by Dr. Sindou and others, I prefer to leave residual tumor when the sinus is involved but still patent and treat those patients with observation if there is no grossly visible residual tumor on the magnetic resonance image, or with radiosurgery if the amount of residual tumor is small enough to be treated in this manner. Additionally, when the sinus is occluded I resect it, but may leave some tumor at one or the other end of the sinus resection when there are important veins draining into that partially patent portion of the sinus. The residual tumor is then treated using radiosurgery.

\section{Falcine Meningiomas}

Most meningiomas involving the anterior third of the falx can be removed radically with or without excision of the superior sagittal sinus, depending on whether the sinus is completely occluded, the pattern of venous drainage, and other factors. Most meningiomas of the posterior falx can also be removed completely, provided that they do not involve the sagittal or the straight sinus; when they do and the sinus is still partially patent, I leave residual tumor rather than sacrifice the sinus and then proceed with postoperative radiosurgery if the residual tumor is small enough, or use fractionated radiotherapy if the amount of residual tumor is larger. Again, falcine tumors involving the middle third of the superior sagittal sinus are more problematic. Very frequently, these tumors are bilateral, and accessing them when the sagittal sinus is patent and cannot be removed is problematic because it requires retraction of the primary motor/sensory regions of the brain. With these tumors, I make every effort to remove the tumor, if possible, from one side and try to avoid opening the opposite side to avoid bilateral damage to the brain and/or central veins in this region, which of course is poorly tolerated. If all of the tumor cannot be removed from one side, I personally prefer to not open the opposite side and leave that residual tumor unless the tumor is very soft and the removal of the tumor could be accomplished with minimal brain retraction and without damage to any of the important central draining veins. When there is residual tumor, I prefer observation, knowing that it will probably have to be treated at some point in the future. Radiosurgery, or more frequently, fractionated radiation, depending upon the size of the residual tumor, is used in elderly patients in hopes of minimizing the chances of having to reoperate in these patients.

\section{Sphenoid Wing Meningiomas}

The more lateral pterional meningiomas frequently cannot be removed completely because of involvement of bone. Obviously, we always attempt as radical a removal as possible, with extensive bone drilling including removal of the superior and lateral walls of the orbit and any intraorbital tumor, but we know that the chances of removing all of the bone containing tumor in these en plaque meningiomas is small. Even when we know that the resection is incomplete, however, I prefer to observe these patients rather than treat them with postoperative radiation because usually these tumors are relatively indolent and the time to recurrence is relatively long. Globular tumors of the middle portion of the sphenoid wing can usually be removed completely. The problem occurs with meningiomas of the medial third of the sphenoid wing, which very frequently involve the cavernous sinus. In spite of my early enthusiasm for radical removal of these tumors after a delightful visit to Vinko Dolenc in Ljubljana, it did not take me long to realize that I did not have the skill required to operate safely in the cavernous sinus and that the postoperative morbidity (cranial nerve deficits) resulting from a few of my early attempts was unacceptable. I now routinely leave residual tumor involving the cavernous sinus, although I make every effort to decompress the optic nerve if it is being compressed by tumor so as to leave enough distance (at least 3-4 $\mathrm{mm}$ ) between the optic nerve and the residual tumor to allow radiosurgical treatment of the residual. ${ }^{5}$

Finally, within this general group we could include the specific case of meningiomas that appear to be centered in the anterior clinoid and project more superiorly. Peculiarly, these tumors usually do not involve the cavernous sinus and can be removed completely. I prefer to perform a thorough extradural removal of the clinoid and unroofing of the optic nerve, which facilitates the subsequent intradural removal of the tumor. If, at surgery, it appears that the tumor involves the cavernous sinus, I prefer to leave residual tumor there, but the issue of whether to proceed with postoperative radiosurgery is complicated in these cases because the residual tumor is frequently very close to the optic nerve. Although my radiosurgical colleagues believe that as long as the dose to the optic nerve does not exceed 10 Gy, radiosurgery could be used, I may prefer to observe these tumors rather than risk damage to the optic nerve. ${ }^{7}$

\section{Midline Anterior Fossa Tumors}

Olfactory groove meningiomas, although frequently giant in size, can of course be removed radically in most patients. Occasionally, the tumors have grown back far enough to be intimately adherent to the anterior cerebral arterial complex and-particularly in elderly patients-it may be preferable to leave some residual tumor, which I do not hesitate to do when it appears that the risk of complete removal is too high. That residual tumor can then be postoperatively observed with the likelihood of a second operation in the future, possibly from a more lateral (pterion$\mathrm{al} /$ transsylvian) approach as opposed to the initial midline bifrontal approach that I usually use for most olfactory groove meningiomas. In older patients, it may be preferable to use either radiosurgery or fractionated radiotherapy, depending upon the size of the residual tumor, in an effort to avoid a second operation. I prefer to approach meningiomas of the planum sphenoidale anterolaterally (pterional approach) rather than from the front. Almost always, these tumors can be removed completely. Meningiomas of the tuberculum are more difficult of course because they frequently involve 1 or both optic nerves. Depending on the situation at surgery, I frequently leave some residual tumor rather than risking blindness (which I have produced more frequently than I want to remember) in an attempt to radi- 
cally remove these tumors. When a residual tumor is left, it is frequently left in intimate contact with the optic nerves, and therefore I usually prefer postoperative observation if the amount of tumor remaining is small. When the amount of tumor remaining is larger or the patient is older and we want to avoid another operation at all costs, I refer these patients for external beam radiotherapy.

\section{Sellar and Suprasellar Region Tumors}

There are, of course, a variety of tumors that occur in the sellar and suprasellar regions. Most pituitary adenomas are approached transsphenoidally and unless the resection has been radical, which is frequently not possible for me to accomplish with macroadenomas, I use postoperative fractionated radiation in these patients given the well-known radiosensitivity of these tumors. The controversies related to the treatment of craniopharyngiomas are well known, and suffice it to say that I am relatively conservative in treating these tumors because I operate only on adults. I suspect that if I were to operate on a child, I would attempt to be more radical, but in adults my policy is one of removing only as much tumor as it appears "safe" to remove without an attempt to be radical. I then use postoperative fractionated radiotherapy in the majority of these patients unless they are older and only a very small amount of tumor has been left, in which case I would prefer observation. Suprasellar meningiomas usually originate from the region of the diaphragm and may be very difficult to remove radically, particularly if the chiasm is prefixed. When I leave residual tumor, which is not infrequent, I would use radiosurgery if the residual tumor is small and at least 3-4 $\mathrm{mm}$ away from the optic nerves. Otherwise, I prefer observation if the residual tumor is small or fractionated radiotherapy if the residual tumor is larger.

\section{Tentorial Incisura Tumors}

Tumors of the lateral incisura can frequently be removed completely. I personally do not hesitate to leave some residual tumor, however, if it appears at surgery that it would be difficult to separate the tumor and preserve the important medial arteries (posterior cerebral, superior cerebellar, and circumferential branches) or nerves, particularly the third cranial nerve. Because reoperation is often not problematic in these patients, I most frequently choose observation of the residual tumor, but in older patients in whom I am hoping to avoid another operation, either radiosurgery or fractionated radiotherapy is recommended depending on the size of the residual tumor. Falcotentorial tumors are another matter and, in my experience, they only can be removed completely when the straight sinus and the vein of Galen are already occluded. I prefer to operate on these tumors only from 1 side to avoid bilateral visual field defects, and therefore I usually end up leaving a significant amount of residual tumor, for which I generally suggest treatment with fractionated radiotherapy in the hope of avoiding another operation in this dangerous region.

\section{Petroclival Tumors}

Petroclival tumors are almost always meningiomas and, unless they are small, it is rare that I am able to accomplish a complete removal. Certainly I do not surgically follow the tumor into the cavernous sinus, and depending upon the consistency of the tumor and its adherence to arterial branches, particularly the small branches of the basilar artery, I prefer to be conservative. It is usually difficult to reoperate in these cases, and therefore I most frequently refer these patients for external beam fractionated radiotherapy in hopes of slowing down the time to recurrence. Epidermoid tumors, which frequently occupy this region, can almost always be removed radically, but I prefer to leave small portions of the capsule if it is adherent to important arteries or nerves. Of course, these patients are never referred for radiation and usually undergo only postoperative observation. My experience has been that these tumors almost invariably recur unless $100 \%$ of the capsule can be removed. The time to symptomatic recurrence is usually 7-15 years, however, and another operation at that time with these tumors is not difficult, although again some of the capsule of the tumor needs to be left, which means that another operation in the future may be necessary.

\section{Tumors of the Meckel Cave}

Most schwannomas of the Meckel cave can be removed radically using a variety of surgical approaches depending upon whether they are in the posterior fossa, the middle fossa, or both (dumbbell-shaped tumors). With schwannomas, I tend to attempt complete removal, even of the portion of the tumor that enters the posterior cavernous sinus; very frequently this can be completed safely with these tumors. Tumors extending into the infratemporal fossa can also generally be removed safely using appropriate skull base techniques. Meningiomas of this region are more difficult to remove completely and frequently a small amount of residual tumor has to be left, which I generally treat using radiosurgery.

\section{Cerebellopontine Angle Tumors}

Most meningiomas of the cerebellopontine angle region can be removed completely, but if a small amount of residual tumor needs to be left to save facial function and hearing, I do not hesitate to do so and then treat the residual portion with postoperative radiosurgery or simply observe the course of the tumor. A more significant change in my attitude has occurred with vestibular schwannomas in this region. With these tumors, the influence of radiosurgery in my practice has been profound; this may be partially related to the fact that I have never been an expert in the surgery of these tumors, and today the standard for preservation of facial and hearing function has been raised substantially. I know very well that in the hands of a few experts with vast experience with these tumors, the rate of facial nerve and hearing preservation with complete excision is comparable to the results of radiosurgery, without subjecting the patient to the miniscule but nevertheless possible risk of developing a malignancy in the future as a result of radiosurgery. I still operate on large tumors causing significant mass effect but do not hesitate to leave some residual tumor if the risk of facial paralysis appears to be too high with an attempt at complete removal. The residual tumor, which is usually 
small, can then be treated with radiosurgery. With smaller tumors that could be treated primarily using radiosurgery, the results in terms of preservation of hearing and facial function in my hands could not compete with the published results of radiosurgery. The issue of long-term tumor control using radiosurgery is gradually being resolved, with several recent publications indicating that when there is tumor regrowth after radiosurgery, it usually occurs during the first few years and that the majority of the patients that experience tumor control for $>10$ years continue to have stable tumors without further growth. In view of these findings, I usually refer patients with small vestibular schwannomas for radiosurgery regardless of age, unless they are elderly, in which case I feel comfortable with observation. With very small intracanalicular tumors I prefer to observe and treat only when there is significant growth.

\section{Posterior Petrosal Tumors}

Meningiomas arising from the posterior face of the petrous bone can usually be removed completely because they push forward the seventh and eighth cranial nerve complex and there is usually a good arachnoid plane between these nerves and the tumor.

\section{Posterior Fossa Tentorial Meningiomas}

Posterior fossa tentorial meningiomas can also usually be removed completely provided they do not involve the venous sinuses; frequently a combined supratentorial and infratentorial approach is required for safe and complete excision of the tentorial insertion of the tumor. Tumors involving the transverse sinus, can usually be removed completely with the sinus provided that a preoperative venogram has shown adequate contralateral venous outflow and the vein of Labbe can be preserved. Tumors involving the sigmoid sinus can also most frequently be removed completely using a skull base approach, provided that there is contralateral patency of venous outflow. Otherwise, some tumor must be left to preserve patency of the involved sinus, and residual tumor can be treated with radiosurgery if it is small enough.

\section{Jugular Foramen Tumors}

Jugular foramen tumors are typically schwannomas, meningiomas, or paragangliomas (glomus jugulare tumors). It is frequently difficult to be able to achieve a safe and complete removal of large meningiomas in this area without damaging the cranial nerves, which in my opinion, is not justifiable. I prefer to leave a small amount of residual tumor that can then be treated using postoperative radiosurgery. Schwannomas can most frequently, but not always, be removed completely with safety using a skull base approach whenever necessary. I do not have significant personal experience with glomus tumors in this location, but currently the majority of them can be removed completely-frequently in collaboration with our colleagues in neurootology and head and neck surgery-after preoperative embolization in large tumors. I remember the days before embolization when these tumors could be devascularized satisfactorily with preoperative low-dose radi- ation therapy, which allowed a safe subsequent excision, and I would not hesitate to recommend this method when embolization is not possible.

\section{Fourth Ventricular Tumors}

Benign choroid plexus papillomas and subependymomas of the fourth ventricle can usually be removed completely without a great deal of difficulty. Hemangioblastomas, of course, are much more difficult surgical tumors, but their treatment method is complete excision, which can most frequently be accomplished with a careful microsurgical technique after devascularizing the larger tumors with preoperative embolization. I do not believe we have enough data to support the use of radiosurgery in these tumors when they cannot be removed safely or as an alternative to surgery when the tumor is located in the brainstem as they usually are.

\section{Foramen Magnum Tumors}

Benign tumors of the foramen magnum region are usually meningiomas, and it is rare to be unable to completely remove them, frequently using a far lateral approach to be able to expose the tumor from a more lateral direction. ${ }^{3}$ Even when the vertebral artery appears to be encased by tumor, it can frequently be dissected from the tumor because of the preservation of an arachnoid plane. Occasionally, however, the vertebral artery and/or cranial nerves are involved in such a way that the risk of complete removal may be too high. Again, I do not hesitate to leave residual tumor in these cases, which can then be observed, particularly in younger patients who would tolerate without difficulty another operation in the future or it can be treated with radiosurgery in hopes of avoiding such an operation, particularly in older patients.

\section{Conclusions}

It is obvious that the recurrent theme of this editorial is that modern forms of radiation therapy, such as radiosurgery (Gamma Knife, linear accelerator, CyberKnife, proton beam, and heavy particle), and more focal and conformal methods of radiation therapy, such as image modulated radiotherapy, as well as the use of fractionated radiosurgery have allowed this surgeon to be more conservative in his approach to benign tumors. The readers must not forget, however, that the surgical treatment of these tumors is highly dependent upon the experience and technical finesse of the surgeon. The generally conservative surgical approach to these tumors outlined earlier is reasonable for surgeons such as myself with considerable microsurgical experience but without a legitimate claim of surgical virtuosity in attacking these different lesions, particularly those involving the skull base. Clearly, a more radical approach is obviously justifiable in the hands of renowned surgeons who have dedicated their careers to conquering the significant surgical challenges presented by these lesions. It is not to them that this editorial is addressed, but rather to the more "general" neurosurgeon that may find some comfort in knowing that one of their experienced colleagues does not hesitate to "back off" and leave residual tumor in patients 


\section{Modern radiation techniques for nonmalignant intracranial tumors}

with difficult benign intracranial tumors. Fortunately, modern radiation techniques and the widespread availability of noninvasive, highly accurate imaging techniques for follow-up have made such a conservative attitude reasonable, at least in my opinion.

\section{References}

1. Friedman WA, Murad GJ, Bradshaw P, Amdur RJ, Mendenhall $\mathrm{WM}$, Foote KD, et al: Linear accelerator surgery for meningiomas. J Neurosurg 103:206-209, 2005

2. Guthrie BL, Carabell SC, Laws ER Jr: Radiation therapy for intracranial meningiomas, in Al-Mefty O (ed): Meningiomas. New York: Raven Press, 1991, pp 255-262

3. Heros RC: Lateral suboccipital approach for vertebral and vertebrobasilar artery lesions. J Neurosurg 64:559-562, 1986

4. Heros RC: Meningiomas involving the sinus. J Neurosurg 105:511-513, 2006
5. Heros RC: Radiosurgery and neurosurgeons. J Neurosurg 103:203-205, 2005

6. Sindou MP, Alvernia JE: Results of attempted radical tumor removal and venous repair in 100 consecutive meningiomas involving the major dural sinuses. J Neurosurg 105:514-525, 2006

7. Steiner L, Lindquist C, Steiner M: Meningiomas and Gamma Knife radiosurgery, in Al-Mefty O (ed): Meningiomas. New York: Raven Press, 1991, pp 263-272

Manuscript submitted March 6, 2008.

Accepted March 6, 2008.

Address correspondence to: Roberto C. Heros, M.D., Department of Neurological Surgery, University of Miami School of Medicine, 1501 NW 9th Avenue, Miami, Florida. email: rheros@med.miami. edu. 